\title{
Filtered Dark Matter at a First Order Phase Transition
}

\author{
Michael J. Baker® ${ }^{1,2, *}$ Joachim Kopp, ${ }^{3,4, \dagger}$ and Andrew J. Long $\oplus^{5, *}$ \\ ${ }^{1}$ School of Physics, The University of Melbourne, Victoria 3010, Australia \\ ${ }^{2}$ Physik-Institut, Universität Zürich, 8057 Zürich, Switzerland \\ ${ }^{3}$ Theoretical Physics Department, CERN, 1211 Geneva, Switzerland \\ ${ }^{4}$ PRISMA Cluster of Excellence and Mainz Institute for Theoretical Physics, Johannes Gutenberg University, \\ Staudingerweg 7, 55099 Mainz, Germany \\ ${ }^{5}$ Rice University, Houston, Texas 77005, USA
}

(Received 23 January 2020; revised 21 April 2020; accepted 16 September 2020; published 9 October 2020)

\begin{abstract}
We describe a new mechanism of dark matter production. If dark matter particles acquire mass during a first order phase transition, it is energetically unfavorable for them to enter the expanding bubbles. Instead, most of them are reflected and quickly annihilate away. The bubbles eventually merge as the phase transition completes and only the dark matter particles that have entered the bubbles survive to constitute the observed dark matter today. This mechanism can produce dark matter with masses from the TeV scale to above the PeV scale, surpassing the Griest-Kamionkowski bound.
\end{abstract}

DOI: 10.1103/PhysRevLett.125.151102

Introduction.-A wealth of observational evidence reveals that the Universe is permeated with a mysterious substance known as dark matter (DM) [1]. Very little, however, is known about the particle physics nature of DM or its origin in the early Universe. Historically, the favored scenario for DM production has been thermal relic production [2-4]. If a DM particle is thermalized with the standard model (SM) plasma in the early Universe then the cosmological expansion, which causes the plasma to cool adiabatically, will eventually make the DM's interactions with the SM inefficient, driving it out of equilibrium. Consequently, the DM relic abundance is determined when these interactions "freeze out," typically increasing with larger DM mass and decreasing with larger interaction strength. Above $m_{\mathrm{DM}} \sim 100 \mathrm{TeV}$ the required interactions violate unitarity [5-7]. This places an upper bound on the mass of thermally produced DM, known as the GriestKamionkowski (GK) bound.

In this Letter, we propose a new mechanism for generating the DM relic abundance. We propose that DM freeze-out did not result from the gradual cooling of the cosmological plasma, but instead was triggered abruptly by a first order cosmological phase transition (FOPT). During the transition, DM particles acquired a mass and low-momentum particles were "filtered" out of the plasma. We will see that DM filtration provides a viable production mechanism, even for DM with masses above the GK bound.

Published by the American Physical Society under the terms of the Creative Commons Attribution 4.0 International license. Further distribution of this work must maintain attribution to the author(s) and the published article's title, journal citation, and DOI. Funded by SCOAP .
The impact of cosmological phase transitions on DM has been studied in a variety of different contexts [8]: a phase transition may alter the expansion rate of the Universe during freeze-out [9-11], inject entropy [10-12], alter DM stability [13-15], alter DM properties during freezein $[16,17]$ (see also [18]), produce DM nonthermally [19-22], or produce an excess of DM over antimatter [23-29]. Conversely, a dark sector may trigger an electroweak FOPT [30-40]. Freeze-out during a second order phase transition has been studied in Refs. [41-43], and Ref. [43] used domain walls to "sweep away" overabundant magnetic monopoles.

Reference [12] recently studied a model where DM acquires mass during a strongly supercooled FOPT and its relic abundance was suppressed by the associated entropy injection. By contrast, our interest is in the dynamical interaction of DM particles with bubble walls and its impact on the relic abundance.

The mechanism.-Our proposed mechanism for DM filtration during a FOPT is illustrated in Fig. 1. DM particles $\chi$ initially have a small mass $m_{\chi}^{\text {out }} \sim T$ and are in thermal equilibrium with SM particles and a new scalar particle $\phi$. We imagine that $\phi$ undergoes the FOPT at temperature $T_{n}$ : its thermal expectation value is initially vanishing, $\langle\phi\rangle=0$, but jumps to a nonzero value, $\langle\phi\rangle=v_{\phi}^{\text {in }}$, during the FOPT. FOPTs proceed through the nucleation and growth of bubbles of the new $\langle\phi\rangle=v_{\phi}^{\text {in }}$ phase [44]. These bubbles expand and merge until the whole Universe has transitioned. At the interface of the old and new phase there is a bubble wall where $\langle\phi\rangle$ smoothly transitions from zero to $v_{\phi}^{\text {in }}$.

We assume that $\langle\phi\rangle \neq 0$ generates a large mass for the DM particles, so light DM particles become heavy as they cross 


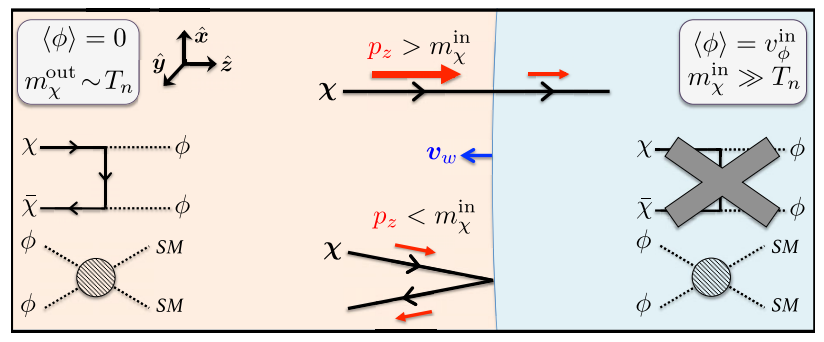

FIG. 1. "Filtered DM": only DM particles $\chi$ with kinetic energy $E \gtrsim m_{\chi}^{\text {in }}$ can penetrate the bubble; slower particles are reflected. In front of the bubble wall (pink region), DM is kept in thermal equilibrium through $\chi \bar{\chi} \leftrightarrow \phi \phi$, but this reaction is put abruptly out of equilibrium at the wall where $\chi$ obtains a mass (blue region). The field $\phi$ remains in equilibrium throughout.

the wall into the bubble. Energy conservation implies that a DM particle can only penetrate the bubble wall if its kinetic energy $E \gtrsim m_{\chi}^{\text {in }}$. Lower momentum modes are reflected by the advancing bubble walls. If $m_{\chi}^{\text {in }} \gg T$, then only an exponentially small fraction of the DM particles will have enough kinetic energy to enter the bubbles. As DM particles enter the bubble, their interactions are put abruptly out of equilibrium, preventing their annihilation. DM particles outside the bubble, in contrast, will continue to interact efficiently, so that the reflected particles quickly annihilate away into the thermal bath. Once the broken phase permeates the whole Universe, only the particles that have entered the bubbles remain and constitute the DM observed today.

A toy model.-To derive quantitative results, we introduce a toy model, which is a viable theory of DM in its own right. We augment the SM by a gauge-singlet real scalar field $\phi(x)$ and a singlet Dirac spinor field $\chi(x)$. The Lagrangian defining this theory contains the terms

$$
\mathcal{L} \supset-V(\phi)-y_{\chi} \phi \bar{\chi} \chi-\beta \phi^{2} H^{\dagger} H,
$$

where $V(\phi)$ is the scalar potential, $y_{\chi}$ is a real Yukawa coupling, $\beta$ is a real Higgs portal coupling, and $H(x)$ is the SM Higgs field. We do not assume any particular form for $V(\phi)$, only that it gives $\phi$ a mass $m_{\phi}$ and causes a FOPT in which $\phi$ acquires a nonzero vacuum expectation value $\langle 0|\phi| 0\rangle=v_{\phi}$. Typically, $v_{\phi}^{\text {in }} \lesssim v_{\phi}$. For simplicity, we assume that the mass of $\phi$ does not change appreciably during the FOPT. Note that $\chi$ enjoys a global U(1) symmetry that ensures its stability.

Before the FOPT, the Yukawa interaction leads to a thermal mass for $\chi, m_{\chi}^{\text {out }}=y_{\chi} T / 4$, while afterward it also induces a larger mass $m_{\chi}^{\text {in }} \sim y_{\chi} v_{\phi}^{\text {in }}$ (we are interested in regimes where $\left.y_{\chi} v_{\phi}^{\text {in }} \gg T_{n} \sim m_{\phi}\right)$. The Yukawa interaction allows $\chi$ to annihilate, chiefly via $\chi \bar{\chi} \rightarrow \phi \phi$, while the thermal mass typically forbids the process $\chi \bar{\chi} \leftrightarrow \phi$. In the following, we retain this condition but otherwise approximate $m_{\chi}^{\text {out }}=0$. We treat $v_{\phi}^{\text {in }} / T_{n}$ as a free parameter, since we do not specify the form of $V(\phi)$, but we remark that large order parameters may arise from nearly conformal potentials
[45-47] or models with heavy fermions (such as $\chi$ here) $[48,49]$.

The Higgs portal interaction [50-52] in Eq. (1) allows the hidden sector to communicate with the SM, through reactions such as $\phi \phi \leftrightarrow H^{\dagger} H$ if $m_{\phi}$ is above the Higgs mass $m_{h}$, and $\phi \phi \leftrightarrow f \bar{f}$ if not. We ensure that $\beta$ is large enough to thermalize $\phi$ and the SM at a common temperature $T_{n}$ during the FOPT. At later times, the Higgs portal interaction allows $\phi$ particles to decay to SM particles. If $m_{\phi}<m_{h} / 2 \simeq 62.5 \mathrm{GeV}$, the Higgs portal coupling is constrained to be $\beta \lesssim 0.007\left(1-4 m_{\phi}^{2} / m_{h}^{2}\right)^{-1 / 4} \quad$ [53], whereas $\beta$ is almost entirely unconstrained if $m_{\phi}>m_{h} / 2$.

A relatively large $m_{\chi}^{\text {in }}$ ensures that $\chi \bar{\chi} \leftrightarrow \phi \phi$ is out of equilibrium inside the bubble. If this were not the case, $\chi$ would remain in thermal equilibrium through the FOPT and its relic abundance would later be determined by standard thermal freeze-out. We therefore require the thermally averaged annihilation rate $\Gamma$ to be smaller than the cosmological expansion rate $H$ inside the bubble. This leads to the condition

$$
\frac{m_{\chi}^{\text {in }}}{T_{n}} \gtrsim 24-\log \frac{T_{n}}{\mathrm{TeV}}-\frac{3}{2} \log \frac{m_{\chi}^{\text {in }} / T_{n}}{24}+4 \log y_{\chi},
$$

where we have used $H=(\pi / \sqrt{90}) \sqrt{g_{*}} T_{n}^{2} / M_{\mathrm{pl}}$ and $\Gamma=\langle\sigma v\rangle n_{\chi}^{\text {in,eq }}$, with the thermally averaged annihilation cross section $\langle\sigma v\rangle \simeq\left(9 y_{\chi}^{4} T_{n}\right) /\left[64 \pi\left(m_{\chi}^{\text {in }}\right)^{3}\right]$ [3] and the would-be equilibrium abundance $n_{\chi}^{\text {in,eq }}=$ $g_{\chi}\left(m_{\chi}^{\text {in }} T_{n} / 2 \pi\right)^{3 / 2} e^{-m_{\chi}^{\text {in }} / T_{n}} \cdot g_{\chi}=2$ counts the spin states, $g_{*} \simeq 100$ is the effective number of relativistic species, and $M_{\mathrm{pl}} \simeq 2.43 \times 10^{18} \mathrm{GeV}$ is the reduced Planck mass. Since $m_{\chi}^{\text {in }}=y_{\chi} v_{\phi}^{\text {in }}$, Eq. (2) allows $y_{\chi}=O(1)$ and $v_{\phi}^{\text {in }} / T_{n}=$ $O(10)$; smaller $y_{\chi}$ needs larger $v_{\phi}^{\text {in }} / T_{n}$.

Analytic estimates.-We first estimate the DM relic abundance by employing a simplified description of the FOPT dynamics, treating the $\chi$ particles as they interact with the wall as if they were free particles. In other words, we assume that the thickness of the bubble wall $l_{w}$ is much smaller than the DM interaction length $l_{\text {int }}$. Because of energy conservation, the mass increase of $\chi$ particles crossing the wall implies that only high-momentum particles can enter the bubble, while low-momentum ones will be reflected. After a distance $l_{\text {int }}$ these reflected particles will be absorbed back into the thermal bath, so low-momentum $\chi$ particles are filtered out of the plasma by the wall. Both reflected and penetrating particles transfer momentum to the bubble wall, leading to friction that limits the speed at which the wall advances $v_{w}[54,55]$.

Using energy and transverse momentum conservation, we find that a massless $\chi$ particle that is incident on the wall with momentum $\mathbf{p}=\left(p_{x}, p_{y}, p_{z}\right)$ (in the plasma's rest frame) will only have sufficient energy to enter the bubble if $\gamma_{w}\left(p_{z}+v_{w}|\mathbf{p}|\right)>m_{\chi}^{\text {in }}$ [56], where $\gamma_{w}=1 / \sqrt{1-v_{w}{ }^{2}}$ is the wall's Lorentz factor and we have assumed the wall 
moves in the negative $z$ direction. Once such a particle enters the bubble, it slows down to travel with a speed $v_{\chi}^{\text {in }}=\left[|\mathbf{p}|^{2}-\left(m_{\chi}^{\text {in }}\right)^{2}\right]^{1 / 2} / m_{\chi}^{\text {in }}$. We will be interested in nonrelativistic walls $v_{w} \lesssim 0.1$ because of the aforementioned friction effect. Moreover, if the wall moves relativistically, most $\chi$ particles enter the bubble.

If a thermal flux of $\chi$ particles is incident on the wall, the number density $n_{\chi}^{\text {in }}$ of $\chi$ particles that have entered the bubble is

$$
\begin{aligned}
n_{\chi}^{\text {in }} & =n_{\bar{\chi}}^{\text {in }}=g_{\chi} \int \frac{d^{3} \mathbf{p}}{(2 \pi)^{3}} \frac{\Theta\left(p_{z}+v_{w}|\mathbf{p}|-m_{\chi}^{\text {in }} / \gamma_{w}\right)}{e^{\mid \mathbf{p} / / T_{n}}+1} \frac{1}{v_{\chi}^{\text {in }}} \\
& \approx \frac{g_{\chi}\left(m_{\chi}^{\text {in }} T_{n}\right)^{3 / 2}}{4(2 \pi)^{3 / 2}} e^{-m_{\chi}^{\text {in }} / T_{n}}=\frac{1}{4} n_{\chi}^{\text {in,eq }},
\end{aligned}
$$

where the step function $\Theta$ enforces the kinematic condition above, $n_{\chi}^{\text {in,eq }}$ was defined below Eq. (2), and $1 / v_{\chi}^{\text {in }}$ accounts for the reduced speed of particles inside the bubble. The Boltzmann-like exponential factor is crucial in suppressing the abundance of DM inside the bubbles and therefore in setting the relic abundance. In front of the bubble wall, reflected DM annihilates $\chi \bar{\chi} \rightarrow \phi \phi$, and $\phi$ remains in equilibrium. The associated entropy transfer and heating are negligible if $g_{*}=O(100)$.

Since $\chi \bar{\chi} \leftrightarrow \phi \phi$ is out of equilibrium inside the bubble, the $\chi$ and $\bar{\chi}$ particles that enter during the phase transition will survive until today, where they constitute the relic population of DM. The corresponding relic abundance $\Omega_{\mathrm{DM}}$ is calculated by scaling $n_{\chi}^{\text {in }}+n_{\bar{\chi}}^{\text {in }}$ with the entropy density $s=\left(2 \pi^{2} / 45\right) g_{* S} T^{3}$, where $g_{* S}=g_{*}$ at $T_{n}$ and $g_{* S}=g_{* S 0} \equiv 3.9$ today (see also Ref. [57]). After normalizing to the critical density $\rho_{c}=3 H_{0}^{2} M_{\mathrm{pl}}^{2}$, we obtain

$$
\begin{aligned}
\Omega_{\mathrm{DM}} h^{2} & \simeq \frac{m_{\chi}\left(n_{\chi}^{\mathrm{in}}+n_{\bar{\chi}}^{\mathrm{in}}\right)}{3 M_{\mathrm{pl}}^{2}\left(H_{0} / h\right)^{2}} \frac{g_{* S 0} T_{0}^{3}}{g_{* S} T_{n}^{3}} \\
& \simeq 0.17\left(\frac{T_{n}}{\mathrm{TeV}}\right)\left(\frac{m_{\chi}^{\text {in }} / T_{n}}{30}\right)^{5 / 2} \frac{e^{-m_{\chi}^{\text {in }} / T_{n}}}{e^{-30}},
\end{aligned}
$$

where $H_{0}=100 \mathrm{~h} \mathrm{~km} / \mathrm{sec} / \mathrm{Mpc}$ is the Hubble constant and $T_{0} \simeq 0.235 \mathrm{meV}$ is the temperature of the cosmic microwave background today. In obtaining this estimate, we have neglected the heating of the SM bath by the annihilation of the reflected $\chi$ particles in front of the wall and by the eventual decay of $\phi$. This is justified because the number of SM degrees of freedom at $T_{n} \gtrsim \mathrm{GeV}$ is much larger than the number of dark sector degrees of freedom. The observed DM relic abundance, $\Omega_{\mathrm{DM}}^{\mathrm{obs}} h^{2} \simeq 0.12$ [58], is obtained if the DM mass increases to $m_{\chi}^{\text {in }} \sim 30 T_{n}$ inside the bubble for $T_{n} \sim 1 \mathrm{TeV}$. At higher (lower) phase transition temperatures, the required $m_{\chi}^{\text {in }} / T_{n}$ becomes larger (smaller), but only logarithmically due to the exponential suppression. Comparing Eq. (4) against the standard thermal freeze-out calculation, we note that our predicted relic abundance only depends on the DM's interaction strength $y_{\chi}$ through $m_{\chi}^{\text {in }} / T_{n}=y_{\chi} v_{\chi}^{\text {in }} / T_{n}$, and consequently, there is a not a one-to-one mapping from the parameters that set the relic abundance to the parameters probed, for instance, by direct detection experiments.

Inside the bubbles, DM could be produced from freeze-in $[12,59,60]$, but for typical parameters $\Omega_{\gamma} h^{2} \sim 10^{-6}$ $\left(y_{\gamma} / 2\right)^{4} e^{-2\left(m_{\chi}^{\text {in }} / T_{n}-32\right)}$, making this population negligible.

Numerical solution of Boltzmann equation.-To obtain a more accurate estimate of the relic abundance, we numerically solve the Boltzmann equations describing the $\chi$ particles near the bubble wall (see Supplemental Material [61]).

Since the scattering and diffusion length scales are small compared to the curvature scale of a typical bubble, we assume that the bubble wall is planar and take the wall to be perpendicular to the $z$ axis. Since the wall experiences a significant drag force from the scattering of $\chi$ particles, we assume a constant nonrelativistic (terminal) wall speed $v_{w}$. We choose $v_{w}=0.01$, but have checked that the final relic abundance is not strongly dependent on its precise value. We approximate the mass profile of DM particles across the wall with a smoothed step function, $m_{\chi}(z)=\frac{1}{2} m_{\chi}^{\text {in }}\left[1+\tanh \left(3 z / l_{w}\right)\right]$. Here and in the remainder of the Letter, we work in the wall's rest frame. We use a wall thickness $l_{w}=1 /\left(4 T_{n}\right)$, but find that the final relic abundance does not depend strongly on the precise value.

Let $f_{a}(t, \mathbf{x}, \mathbf{p})$ be the phase space distribution functions for $a=\chi, \bar{\chi}$, and $\phi$ particles. We assume that the conserved $\chi-\bar{\chi}$ asymmetry is vanishing, thus $f_{\bar{\chi}}=f_{\chi}$, and that $\phi$ remains in equilibrium throughout the FOPT: $f_{\phi}=f_{\phi}^{\mathrm{eq}}$ is the Bose-Einstein distribution. This is justified provided that $\phi$ depletion is fast enough to keep up with $\phi$ production. Far in front of the wall $(z \rightarrow-\infty), f_{\chi}=f_{\chi}^{\text {eq }}$ follows the FermiDirac distribution. We adopt the ansatz

$$
f_{\chi}(z, \mathbf{p})=\mathcal{A}\left(z, p_{z}\right) \times f_{\chi}^{\mathrm{eq}}(z, \mathbf{p}),
$$

motivated in the Supplemental Material [61]. The distribution $f_{\chi}$ in the vicinity of the bubble wall can then be described by the Boltzmann equation

$$
\left\{\left[\frac{p_{z}}{m_{\chi}} \frac{\partial}{\partial z}-\left(\frac{\partial m_{\chi}}{\partial z}\right) \frac{\partial}{\partial p_{z}}-\left(\frac{\partial m_{\chi}}{\partial z}\right) \frac{v_{w}}{T_{n}}\right] \mathcal{A}\left(z, p_{z}\right)\right\} \frac{g_{\chi} m_{\chi} T_{n}}{2 \pi} \exp \left(\frac{v_{w} p_{z}-\sqrt{m_{\chi}^{2}+\left(p_{z}\right)^{2}}}{T_{n}}\right)=g_{\chi} \int \frac{d p_{x} d p_{y}}{(2 \pi)^{2}} \mathbf{C}\left[f_{\chi}\right] .
$$




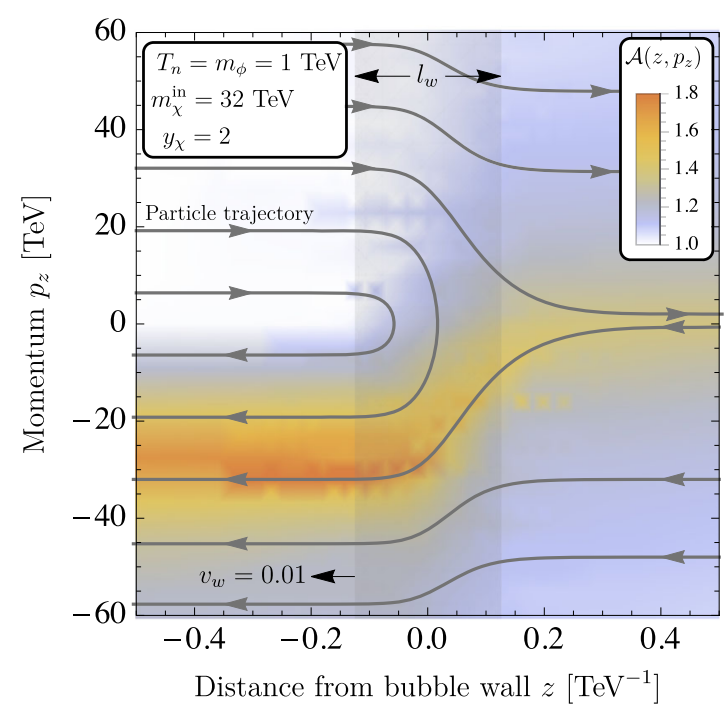

FIG. 2. The enhancement factor $\mathcal{A}\left(z, p_{z}\right)$ in the neighborhood of the bubble wall (opaque vertical band). Contours with arrows indicate possible particle trajectories in this two-dimensional phase space. For the chosen parameter values, we recover the observed relic abundance.

The right-hand side includes the collision terms for the processes $\chi \bar{\chi} \rightarrow \phi \phi, \chi \phi \rightarrow \chi \phi, \chi \chi \rightarrow \chi \chi$, and $\chi \bar{\chi} \rightarrow \chi \bar{\chi}$. Note that we have integrated over $p_{x}$ and $p_{y}$. Integrating over $p_{z}$ will then yield the number density at a position $z$.

We are interested in solutions of Eq. (6) that obey the boundary conditions

$$
\lim _{\substack{z \rightarrow-\infty \\ p_{z}>0}} \mathcal{A} \rightarrow 1 \text { and } \quad \lim _{z \rightarrow \infty} \mathcal{A}\left(p_{z}\right)=\lim _{z \rightarrow \infty} \mathcal{A}\left(-p_{z}\right) .
$$

The first condition enforces an equilibrium phase space distribution for particles that have not yet interacted with the bubble wall, while the second condition is based on the assumption that at a large positive $z$ the other side of the bubble is advancing with similar dynamics. We solve Eqs. (6) and (7) numerically using the method of characteristics, where the two-dimensional partial differential equation is rewritten as an infinite set of uncoupled ordinary differential equations. Each equation corresponds to a possible particle trajectory in the two-dimensional phase space spanned by $z$ and $p_{z}$, in the absence of collisions. A typical solution for $\mathcal{A}\left(z, p_{z}\right)$ is shown in Fig. 2, along with some of the aforementioned particle trajectories. Particles incident on the wall begin in equilibrium (upper-left quadrant), so $\mathcal{A}\left(z, p_{z}\right) \approx 1$. Those that started with a momentum larger than $m_{\chi}^{\text {in }}$ enter the bubble (upper right), with $\mathcal{A} \approx 1.2$. That is, with an abundance only slightly larger than the strongly Boltzmann-suppressed $f_{\chi}^{\mathrm{eq}}(z, \mathbf{p})$. Particles that started with a momentum lower than $m_{\chi}^{\text {in }}$ are reflected by the wall (midleft). Particles that come from $z \rightarrow \infty$ (lower right) receive a boost in momentum as they leave the bubble. These boosted particles and the reflected particles lead to an overdensity, which annihilates into the

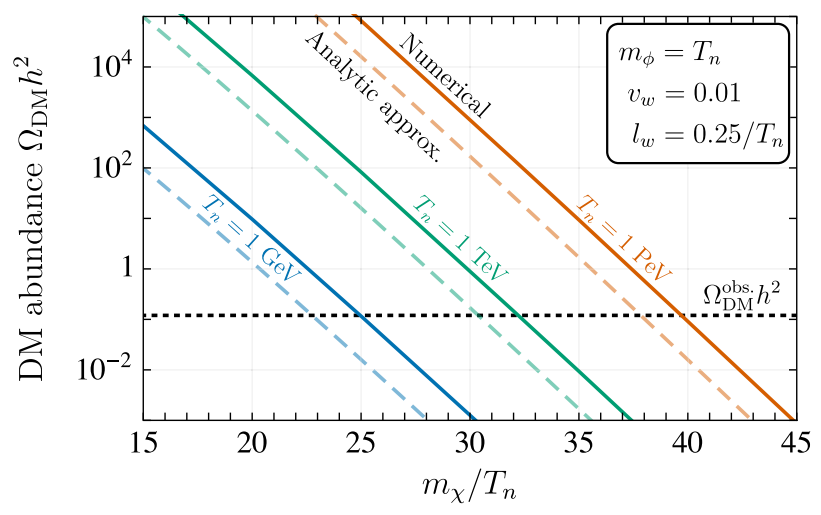

FIG. 3. The DM relic abundance as a function of the FOPT's temperature $T_{n}$ and the $\chi$ particle's mass $m_{\chi}$, where we assume $m_{\chi} \approx m_{\chi}^{\text {in }}$. The solid lines are calculated by numerically solving the Boltzmann equation, while the dashed lines show the analytic approximation (4).

thermal bath as the particles travel away from the wall (bottom left).

We then integrate over $p_{z}$ deep inside the bubble to find the resulting DM relic abundance and present our results in Fig. 3. We assume $m_{\chi} \approx m_{\chi}^{\text {in }}$, implying a negligible change in the $\chi$ particle's mass between the FOPT and today. The observed relic abundance is obtained for $m_{\chi} / T_{n} \approx$ $(25,32,40)$ and $T_{n}=(1 \mathrm{GeV}, 1 \mathrm{TeV}, 1 \mathrm{PeV})$, respectively. These parameters are consistent with the out-of-equilibrium condition (2) provided that $y_{\chi}<(0.2, \sqrt{4 \pi}, \sqrt{4 \pi})$, respectively. The exponential sensitivity to $m_{\chi} / T_{n} \approx m_{\chi}^{\text {in }} / T_{n}$ is clearly visible. Comparing the numerical result with the analytical estimate from Eq. (4), we find good agreement of the parametric dependences on $T_{n}$ and $m_{\chi}^{\text {in }} / T_{n}$, and the overall amplitude differs by a factor of $\sim 5$.

Current and future probes.-Filtered DM is amenable to many of the same tests as thermal relic (weakly interacting massive particle) DM. Direct detection of $\chi$ particles is mediated, in this toy model, via exchange of $\phi$ particles and Higgs bosons $(h)$, so the rate is suppressed by the tiny $\phi-h$ mixing [62]. In Fig. 4, the purple region shows the range of spin-independent $\chi$-nucleon scattering cross sections $\sigma_{\chi^{N}}^{\mathrm{SI}}$. We impose the conditions that $\Omega_{\chi}=\Omega_{\mathrm{DM}}^{\mathrm{obs}}$, couplings remain perturbative $\left(y_{\chi}, \beta<\sqrt{4 \pi}\right), \chi$ is in equilibrium outside the bubble and out of equilibrium inside the bubble, Eq. (2), and $\phi$ is in equilibrium throughout the FOPT. At $m_{\chi} \ll 100 \mathrm{GeV}$, the dark sector no longer stays in equilibrium outside the bubble because $\phi \phi$ annihilation is suppressed by the Higgs mass and small SM Yukawa couplings. Around masses of several TeV, the value of $\beta$ required to keep $\phi$ in equilibrium grows, making it impossible to obtain the correct Higgs mass from the scalar mass matrix. At even larger $m_{\chi}$, this problem disappears as new $\phi$ annihilation channels open up. We see that there is a large region of viable parameter space at masses above the Griest-Kamionkowski bound $[5,6]$. 


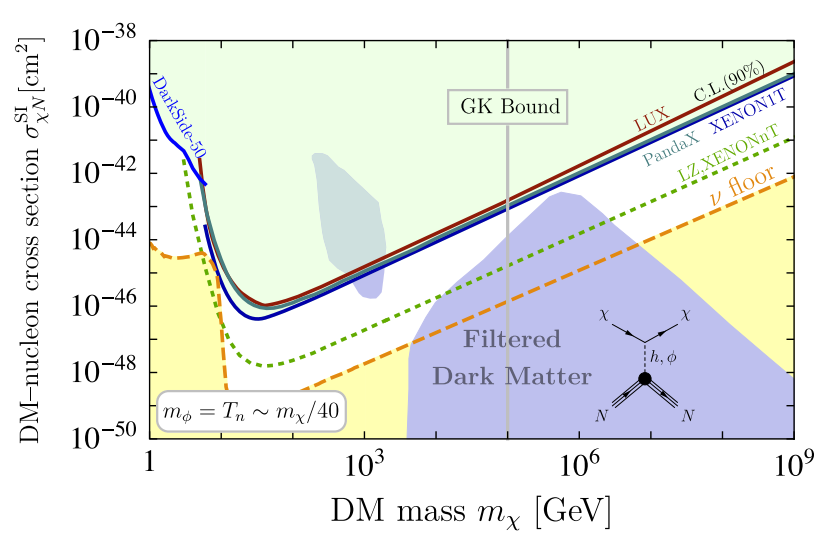

FIG. 4. The predicted spin-independent DM-nucleon scattering cross section (purple shaded region) in comparison with various experimental exclusions limits (green shaded) [63-66], projected sensitivities of future experiments (green dashed) [67], and the neutrino floor (yellow shaded). Note that viable models of filtered DM are obtained even at DM masses above the Griest-Kamionkowski bound, $m_{\chi} \sim 100 \mathrm{TeV}$.

At current and future collider experiments, filtered DM can be tested through precision measurements of the Higgs boson's couplings to other SM particles [68-71]. These measurements already constrain the $\phi-h$ mixing for sub$\mathrm{TeV}$ masses [72,73].

Annihilations of $\chi$ and $\bar{\chi}$ to SM particles in the Milky Way's DM halo provide another avenue to indirectly detect filtered DM. Decays of the annihilation products may be a source of PeV-scale neutrinos. Detection prospects are, however, hampered by $p$-wave-suppressed annihilation cross sections.

The FOPT bubble dynamics produce a stochastic background of gravitational waves [74]. The frequency of this radiation is tied to the DM mass scale. However, we expect the signal strength to be suppressed by the small bubble wall speed and a dedicated analysis is required to determine if this signal is within reach of next-generation gravitational wave telescopes, e.g., Laser Interferometer Space Antenna [75].

The authors would like to thank Andrea Thamm for comments on the manuscript and Christopher Tunnell for discussions of direct detection prospects. The authors would also like to express a special thanks to the Mainz Institute for Theoretical Physics (MITP) for its hospitality and support during key parts of the collaboration. M. J. B. was supported by the Australian Research Council and by the Swiss National Science Foundation (SNF) under Contract No. 200021-159720. J. K. has been partially supported by the European Research Council (ERC) under the European Union's Horizon 2020 research and innovation program (Grant Agreement No. 637506, " $\nu$ Directions") and by the German Research Foundation (DFG) under Grant No. KO 4820/4-1. A. J. L. was supported in part by the U.S. Department of Energy under Award No. DE-SC0007859. *michael.baker@unimelb.edu.au

†jkopp@cern.ch ¥andrewjlong@rice.edu

[1] G. Bertone and D. Hooper, History of dark matter, Rev. Mod. Phys. 90, 045002 (2018).

[2] M. Srednicki, R. Watkins, and K. A. Olive, Calculations of relic densities in the early Universe, Nucl. Phys. B310, 693 (1988).

[3] P. Gondolo and G. Gelmini, Cosmic abundances of stable particles: Improved analysis, Nucl. Phys. B360, 145 (1991).

[4] K. Griest and D. Seckel, Three exceptions in the calculation of relic abundances, Phys. Rev. D 43, 3191 (1991).

[5] K. Griest and M. Kamionkowski, Unitarity Limits on the Mass and Radius of Dark Matter Particles, Phys. Rev. Lett. 64, 615 (1990).

[6] I. Baldes and K. Petraki, Asymmetric thermal-relic dark matter: Sommerfeld-enhanced freeze-out, annihilation signals and unitarity bounds, J. Cosmol. Astropart. Phys. 09 (2017) 028.

[7] J. Smirnov and J. F. Beacom, TeV-scale thermal WIMPs: Unitarity and its consequences, Phys. Rev. D 100, 043029 (2019).

[8] D. N. Schramm, Phase transitions and dark matter problems, Nucl. Phys. B252, 53 (1985).

[9] E. W. Kolb and S. Wolfram, Spontaneous symmetry breaking and the expansion rate of the early Universe, Astrophys. J. 239, 428 (1980).

[10] D. Chung, A. Long, and L.-T. Wang, Probing the cosmological constant and phase transitions with dark matter, Phys. Rev. D 84, 043523 (2011).

[11] D. J. H. Chung and A. J. Long, Cosmological constant, dark matter, and electroweak phase transition, Phys. Rev. D 84, 103513 (2011).

[12] T. Hambye, A. Strumia, and D. Teresi, Super-cool dark matter, J. High Energy Phys. 08 (2018) 188.

[13] M. J. Baker and J. Kopp, Dark Matter Decay between Phase Transitions at the Weak Scale, Phys. Rev. Lett. 119, 061801 (2017).

[14] M. J. Baker, Dark matter models beyond the WIMP paradigm, Nuovo Cimento Soc. Ital. Fis. 40C, 163 (2017).

[15] M. J. Baker and L. Mittnacht, Variations on the Vev flipflop: Instantaneous freeze-out and decaying dark matter, J. High Energy Phys. 05 (2019) 070.

[16] M. J. Baker, M. Breitbach, J. Kopp, and L. Mittnacht, Dynamic freeze-in: Impact of thermal masses and cosmological phase transitions on dark matter production, J. High Energy Phys. 03 (2018) 114.

[17] L. Bian and Y.-L. Tang, Thermally modified sterile neutrino portal dark matter and gravitational waves from phase transition: The freeze-in case, J. High Energy Phys. 12 (2018) 006.

[18] T. Cohen, D. E. Morrissey, and A. Pierce, Changes in dark matter properties after freeze-out, Phys. Rev. D 78, 111701 (2008).

[19] E. Witten, Cosmic separation of phases, Phys. Rev. D 30, 272 (1984).

[20] A. Falkowski and J. M. No, Non-thermal dark matter production from the electroweak phase transition: MultiTeV WIMPs and "Baby-Zillas", J. High Energy Phys. 02 (2013) 034. 
[21] F. P. Huang and C. S. Li, Probing the baryogenesis and dark matter relaxed in phase transition by gravitational waves and colliders, Phys. Rev. D 96, 095028 (2017).

[22] Y. Bai, A.J. Long, and S. Lu, Dark quark nuggets, Phys. Rev. D 99, 055047 (2019).

[23] S. Dodelson, B. R. Greene, and L. M. Widrow, Inverse phase transitions: Does baryogenesis lead to dark matter?, in 15th Johns Hopkins Workshop on Current Problems in Particle Theory: Particle Physics from Underground to Heaven Baltimore, Maryland, 1991 (World Scientific, Singapore, 1992), pp. 391-402, https://cds.cern.ch/record/ 238229.

[24] J. Shu, T. M.P. Tait, and C.E. M. Wagner, Baryogenesis from an earlier phase transition, Phys. Rev. D 75, 063510 (2007).

[25] K. Petraki, M. Trodden, and R. R. Volkas, Visible and dark matter from a first-order phase transition in a baryonsymmetric universe, J. Cosmol. Astropart. Phys. 02 (2012) 044.

[26] I. Baldes, Gravitational waves from the asymmetric-darkmatter generating phase transition, J. Cosmol. Astropart. Phys. 05 (2017) 028.

[27] B. Fornal, Y. Shirman, T. M. P. Tait, and J. R. West, Asymmetric dark matter and baryogenesis from $\mathrm{SU}(2)_{\ell}$, Phys. Rev. D 96, 035001 (2017).

[28] P.-H. Gu, Cosmic matter from dark electroweak phase transition with neutrino mass generation, Phys. Rev. D 96, 055038 (2017).

[29] E. Hall, T. Konstandin, R. McGehee, and H. Murayama, Asymmetric matters from a dark first-order phase transition, arXiv:1911.12342.

[30] M. Gonderinger, Y. Li, H. Patel, and M. J. Ramsey-Musolf, Vacuum stability, perturbativity, and scalar singlet dark matter, J. High Energy Phys. 01 (2010) 053.

[31] M. Carena, N. R. Shah, and C. E. M. Wagner, Light dark matter and the electroweak phase transition in the NMSSM, Phys. Rev. D 85, 036003 (2012).

[32] T. A. Chowdhury, M. Nemevsek, G. Senjanovic, and Y. Zhang, Dark matter as the trigger of strong electroweak phase transition, J. Cosmol. Astropart. Phys. 02 (2012) 029.

[33] D. Borah and J. M. Cline, Inert doublet dark matter with strong electroweak phase transition, Phys. Rev. D 86, 055001 (2012).

[34] G. Gil, P. Chankowski, and M. Krawczyk, Inert dark matter and strong electroweak phase transition, Phys. Lett. B 717, 396 (2012).

[35] M. Fairbairn and R. Hogan, Singlet fermionic dark matter and the electroweak phase transition, J. High Energy Phys. 09 (2013) 022.

[36] A. Ahriche and S. Nasri, Dark matter and strong electroweak phase transition in a radiative neutrino mass model, J. Cosmol. Astropart. Phys. 07 (2013) 035.

[37] T. Alanne, K. Tuominen, and V. Vaskonen, Strong phase transition, dark matter and vacuum stability from simple hidden sectors, Nucl. Phys. B889, 692 (2014).

[38] W. Chao, H.-K. Guo, and J. Shu, Gravitational wave signals of electroweak phase transition triggered by dark matter, J. Cosmol. Astropart. Phys. 09 (2017) 009.

[39] P. H. Ghorbani, Electroweak baryogenesis and dark matter via a pseudoscalar vs. scalar, J. High Energy Phys. 08 (2017) 058.
[40] K. Ghorbani and P. H. Ghorbani, A simultaneous study of dark matter and phase transition: Two-scalar scenario, J. High Energy Phys. 12 (2019) 077.

[41] S. Dimopoulos, R. Esmailzadeh, L. J. Hall, and N. Tetradis, Electroweak phase transition and dark matter abundance, Phys. Lett. B 247, 601 (1990).

[42] Lucien Heurtier and Herve Partouche, Spontaneous freeze out of dark matter from an early thermal phase transition, Phys. Rev. D 101, 043527 (2020).

[43] G. R. Dvali, H. Liu, and T. Vachaspati, Sweeping Away the Monopole Problem, Phys. Rev. Lett. 80, 2281 (1998).

[44] A. D. Linde, Decay of the false vacuum at finite temperature, Nucl. Phys. B216, 421 (1983); Erratum, Nucl. Phys. B223, 544 (1983).

[45] P. Creminelli, A. Nicolis, and R. Rattazzi, Holography and the electroweak phase transition, J. High Energy Phys. 03 (2002) 051.

[46] G. Nardini, M. Quiros, and A. Wulzer, A confining strong first-order electroweak phase transition, J. High Energy Phys. 09 (2007) 077.

[47] T. Konstandin and G. Servant, Cosmological consequences of nearly conformal dynamics at the TeV scale, J. Cosmol. Astropart. Phys. 12 (2011) 009.

[48] M. Carena, A. Megevand, M. Quiros, and C. E. M. Wagner, Electroweak baryogenesis and new $\mathrm{TeV}$ fermions, Nucl. Phys. B716, 319 (2005).

[49] A. Angelescu and P. Huang, Multistep strongly first order phase transitions from new fermions at the $\mathrm{TeV}$ scale, Phys. Rev. D 99, 055023 (2019).

[50] V. Silveira and A. Zee, Scalar phantoms, Phys. Lett. 161B, 136 (1985).

[51] C. P. Burgess, M. Pospelov, and T. ter Veldhuis, The minimal model of nonbaryonic dark matter: A singlet scalar, Nucl. Phys. B619, 709 (2001).

[52] B. Patt and F. Wilczek, Higgs-field portal into hidden sectors, arXiv:hep-ph/0605188.

[53] A. M. Sirunyan et al. (CMS Collaboration), Search for invisible decays of a Higgs boson produced through vector boson fusion in proton-proton collisions at $\sqrt{s}=13 \mathrm{TeV}$, Phys. Lett. B 793, 520 (2019).

[54] G. D. Moore and T. Prokopec, Bubble Wall Velocity in a First Order Electroweak Phase Transition, Phys. Rev. Lett. 75, 777 (1995).

[55] G. D. Moore and T. Prokopec, How fast can the wall move? A study of the electroweak phase transition dynamics, Phys. Rev. D 52, 7182 (1995).

[56] D. Bodeker and G. D. Moore, Can electroweak bubble walls run away?, J. Cosmol. Astropart. Phys. 05 (2009) 009.

[57] Y. Nakai, N. Shiba, and M. Yamada, Entanglement entropy and decoupling in the Universe, Phys. Rev. D 96, 123518 (2017).

[58] N. Aghanim et al. (Planck Collaboration), Planck 2018 results. VI. Cosmological parameters, Astron. Astrophys. 641, A6 (2020).

[59] J. McDonald, Thermally Generated Gauge Singlet Scalars as Self-Interacting Dark Matter, Phys. Rev. Lett. 88, 091304 (2002).

[60] L. J. Hall, K. Jedamzik, J. March-Russell, and S. M. West, Freeze-in production of FIMP dark matter, J. High Energy Phys. 03 (2010) 080. 
[61] See Supplemental Material at http://link.aps.org/supplemental/ 10.1103/PhysRevLett.125.151102 for derivation and solution of the relevant Boltzmann equations.

[62] S. Matsumoto, Y.-L.S. Tsai, and P.-Y. Tseng, Light fermionic WIMP dark matter with light scalar mediator, J. High Energy Phys. 07 (2019) 050.

[63] D. S. Akerib et al. (LUX Collaboration), Results from a Search for Dark Matter in the Complete LUX Exposure, Phys. Rev. Lett. 118, 021303 (2017).

[64] X. Cui et al. (PandaX-II Collaboration), Dark Matter Results from 54-Ton-Day Exposure of PandaX-II Experiment, Phys. Rev. Lett. 119, 181302 (2017).

[65] E. Aprile et al. (XENON Collaboration), Dark Matter Search Results from a One Ton-Year Exposure of XENON1T, Phys. Rev. Lett. 121, 111302 (2018).

[66] P. Agnes et al. (DarkSide Collaboration), Low-Mass Dark Matter Search with the DarkSide-50 Experiment, Phys. Rev. Lett. 121, 081307 (2018).

[67] D. S. Akerib et al. (LUX-ZEPLIN Collaboration), Projected WIMP sensitivity of the LUX-ZEPLIN (LZ) dark matter experiment, Phys. Rev. D 101, 052002 (2020).

[68] K. Fujii et al., Physics case for the $250 \mathrm{GeV}$ stage of the international linear collider, arXiv:1710.07621.
[69] M. Dong et al. (CEPC Study Group Collaboration), CEPC conceptual design report: Volume 2-physics \& detector, arXiv:1811.10545.

[70] A. Abada et al. (FCC Collaboration), FCC physics opportunities, Eur. Phys. J. C 79, 474 (2019).

[71] M. Cepeda et al. (HL/HE WG2 Group Collaboration), Higgs physics at the HL-LHC and HE-LHC, CERN Yellow Rep. Monogr. 7, 221 (2019).

[72] G. Aad et al. (ATLAS and CMS Collaborations), Measurements of the Higgs boson production and decay rates and constraints on its couplings from a combined ATLAS and CMS analysis of the LHC $p p$ collision data at $\sqrt{s}=7$ and $8 \mathrm{TeV}$, J. High Energy Phys. 08 (2016) 045.

[73] M. Carena, Z. Liu, and M. Riembau, Probing the electroweak phase transition via enhanced di-Higgs boson production, Phys. Rev. D 97, 095032 (2018).

[74] M. Kamionkowski, A. Kosowsky, and M. S. Turner, Gravitational radiation from first order phase transitions, Phys. Rev. D 49, 2837 (1994).

[75] C. Caprini et al., Detecting gravitational waves from cosmological phase transitions with LISA: An update, J. Cosmol. Astropart. Phys. 03 (2020) 024. 